\title{
EDIFICATE
}

I Congreso de Escuelas de Edificación y Arquitectura Técnica de España

València, 4 y 5 de noviembre de 2021

Escuela Técnica Superior de Ingeniería de Edificación

Universitat Politècnica de València

Doi: https://doi.org/10.4995/EDIFICATE2021.2021.13531

\section{Investigación aplicada a la formación en nuevos materiales de construcción}

\section{Applied research to new construction materials subject}

\author{
Jesús Gadea Sáinz a , Verónica Calderón Carpinterob ${ }^{b}$ Carlos Junco Petrement c, Sara \\ Gutiérrez-González $^{\mathrm{d}}$, Ángel Rodríguez Saiz ${ }^{\mathrm{e}}$, Javier Garabito López ${ }^{\mathrm{f}}$ \\ Departamento de Construcciones Arquitectónicas e Ingeniería de la Construcción y del Terreno, \\ Escuela Politécnica Superior de la Universidad de Burgos \\ ajgadeamc@ubu.es, bvcalderon@ubu.es, ccjunco@ubu.es, dsggonzalez@ubu.es, \\ earsaizmc@ubu.es, fjgarabito@ubu.es
}

\begin{abstract}
The degree in Building Engineering at the University of Burgos has been included the Bologna System 10 years ago, implemented simultaneously in the 2010-2011 academic year the entire degree - four courses. According with the initial strategies, the acquisition of competences through project research work in the construction materials subjects has been one of the elements developed and consolidated, especially in the elective subjects of higher courses where training of the student already allows this type of resources. However, the work begins the first year with specific classes to introduce the investigation within the subjects of construction materials, with agenda reflected in the educational guide. One of them, for example, is an intensive course of advanced searches in international scientific databases, which must be used in a practical way to test what has been learned, and which can be extrapolated to the rest of the subjects of the degree.
\end{abstract}

Keywords: Project management skills, new construction materials, applied research tools, transferable' skills. 


\section{Resumen}

El grado en Arquitectura Técnica de la Universidad de Burgos lleva un recorrido de 10 años en el Plan Bolonia, que implantó de manera simultánea en el curso 2010-2011 para los cuatro cursos del grado. Dentro de las estrategias iniciales del Grado, la adquisición de competencias a través del trabajo por proyectos en las asignaturas de materiales de construcción ha sido uno de los factores que se ha desarrollado y afianzado, especialmente en la asignaturas optativas de cursos más altos donde la formación del estudiante ya permite este tipo de recursos. No obstante, se comienza desde primer curso con clases específicas de introducción a la investigación dentro de las asignaturas de materiales de construcción, con temario contemplado en la guía docente. Una de ellas, por ejemplo, es un curso internsivo de búsquedas avanzadas en bases de datos científicas internacionales, que debe ser utilizado de manera práctica para poner a prueba lo aprendido, y que puede ser extrapolado al resto de asignaturas de la titulación.

Palabras clave: Competencias de gestión de proyectos, nuevos materiales de construcción, herramientas de investigación aplicada, competencias transferibles. 


\section{Introducción}

La investigación aplicada se utiliza en alguna de las asignaturas de Materiales de Construcción como un proceso pedagógico que complementa el resto de métodos de enseñanza y aprendizaje, siendo especialmente útil en los últimos cursos de la Titulación. También se utiliza para abordar diferentes problemas asociados con los paradigmas de enseñanza y la dinámica del aula para una mejor experiencia de aprendizaje, aplicando muchos de los conocimientos previos adquiridos y otros que se consiguen durante este proceso formativo específico.

Elaborar e implementar un modelo para el desarrollo de la investigación aplicada en carreras técnicas desde la implantación de Bolonia ha sido un factor clave para determinar la calidad del curriculum en la formación teórico-práctica, y en el grado de especialización del mismo. No en vano, el paso de la diplomatura (180 créditos ECTS) a grado (240 créditos ECTS) en los estudios de Arquitectura Técnica supuso un gran desafío, pero también significó significó la apertura y la posibilidad de acceso directo a la carrera investigadora, dado el alcance académico de los estudios de grado en comparación con los títulos técnicos anteriores.

Además de la investigación aplicada dentro del taller y laboratorios de las asignaturas de Materiales de Construcción, se utilizan los entornos virtuales de aprendizaje como recurso didáctico, lo que incluye el empleo, entre otras cosas, de la plataforma educativa UBUVirtual y la biblioteca digital, desde la que se gestionan las bases de datos científicas, buscadores de patentes y registros de propiedad, y normativa nacional e internacional aplicable a los materiales de construcción, fortaleciendo el trabajo teórico y practico para el desarrollo del sector.

Esta innovación docente se enmarca dentro de la formación a través de la investigación basada en el conocimiento y la comprensión de cómo aplicar ese conocimiento en el ejercicio futuro de la profesión. Algunos estudios derivados de la aplicación de los principios propuestos a través de la aplicación aplicada afirman, a través de las conclusiones de sus docentes, que los estudiantes suelen conventirse en egresados con mejores capacidades para resolver problemas inesperados, que se benefician de la evaluación formativa y se vuelven más conscientes del objeto de aprendizaje que luego puede conducir a un mejor traslado profesional.

Promover este tipo de aprendizaje es una manera de impulsar la cultura de investigación en la formación universitaria en titulaciones tradicionalmente técnicas, puesto que se comprueba la utilidad inmediata de la aplicación, al mismo tiempo que se incentiva la capacidad investigadora y crítica. 


\section{Objetivos}

El aprendizaje basado en proyectos es una forma de instrucción centrada en el estudiante que se basa en tres principios: el aprendizaje es específico del contexto, la participación activa del aprendizaje y la interacción social mediante el intercambio de conocimientos. Además de las competencias propias del conocimiento académico y formal de las asignaturas de Materiales de Construcción, se busca alcanzar cinco de las seis categorías de las que se ocupan de las habilidades interpersonales: habilidades de comunicación, habilidades organizativas, habilidades de formación de equipos, habilidades de liderazgo y habilidades para abordar situaciones novedosas.

Por todo lo anterior, el objetivo principal de este proyecto de innovación docente se basa en la aplicación del aprendizaje por proyectos de investigación como respuesta a una demanda y necesidad de las nuevas metodologías en la enseñanza, especialmente en los cursos más avanzados de la titulación.

\section{Metodología}

Las asignaturas de Materiales en la Titulación de Arquitectura Técnica en la Universidad de Burgos se corresponden con el primer, segundo y cuarto curso, y son cuatro: Fundamentos de Materiales (primer curso, primer semestre), Materiales I (primer curso, segundo semestre), Materiales I (segundo curso, primer semestre) y Nuevos Materiales de Aplicación en Construcción (cuarto curso, segundo semeste). Todas ellas son materiales de 6 créditos.

Para poder aplicar los conocimientos previos adquiridos al respecto de estas asignaturas relacionadas con los Materiales de Construcción, se incide específicamente en la innovación docente llevada a cabo en la asignatura de Nuevos Materiales de Aplicación en Construcción. Este curso se presta especialmente a este tipo de aprendizaje, donde se combina la enseñanza propia del temario con la gestión de la investigación aplicada al desarrollo de un proyecto real de materiales novedosos aplicados en obra real. Además, surgen otros problemas inesperados a medida que el proyecto se desarrolla, de modo que la lista de temas siempre se expande más allá del conjunto tradicional.

El curso está estructurado para proporcionar a los estudiantes el conocimiento que será necesario para planificar y gestionar el trabajo de investigación aplicado, incluyendo el aprendizaje de herramientas complementarias como son búsqueda bibliográfica en bases de datos internación.ales, en buscadores mundiales de patentes y modelos de utilidad y en revistas técnicas especializadas.

Además del trabajo colaborativo por grupos generalmente de dos o tres personas, se asignan a cada estudiante tareas individuales, lo que asegura que todos los miembros del equipo dominen el material técnico.

Los estudiantes eligen, bajo supervisión del profesor, el material de construcción novedoso sobre el que van a trabajar, (fase de elección) lo que repercute positivamente en la 
implicación a lo largo del proyecto. Dar asignaciones de proyectos grupales a los estudiantes desarrolla las habilidades sociales de los estudiantes, como por ejemplo el liderazgo, la comunicación, el trabajo en equipo y los problemas habilidades para resolver, además de fortalecer la mentalidad adecuada para enfrentarse a las realidades de la vida al ejecutar proyectos. La identificación de las fuentes fiables y de la búsqueda y empleo de los recursos científico-técnicos, también son partes relevantes del proyecto (fase de preparación y planificación)

Durante el desarrollo del trabajo, también se llevan a cabo prácticas en el laboratorio químico y en el taller de materiales o de grandes estructuras. La finalidad consiste en dotar a los estudiantes de una visión global y multidisciplinar del funcionamiento de un material de construcción, y la incidencia de la microestructura en las propiedades finales a largo plazo. La idea es que ese conocimiento pueda ser trasladado también a cada trabajo concreto de investigación (fase de ejecución).

Una vez concluido el desarrollo colaborativo, se defienden y evalúan los trabajos en clase de manera expositiva. Finalmente, se sacan las conclusiones correspondientes del tema elegido, tanto de manera individual como de manera colectiva en la sesión expositiva, de manera que se decide si la solución tecnológica o científica al problema o proyecto planteado ha sido adecuada (fase de evaluación).

\section{Resultados de la innovación}

Los resultados obtenidos con esta metodología de investigación aplicada, comparados con los del método tradicional, indican que, aunque los estudiantes tienden a adoptar una actitud pasiva y son reacios a aumentar el grado de trabajo autónomo en un principio, el procedimiento contribuye a mejorar la aplicación de los conceptos teóricos en el diseño y la realización del proyecto.

Los estudiantes tienen el desafío de pensar críticamente sobre el trabajo realizado, vincular sus observaciones con los conceptos del curso, y discutir cómo pueden aplicar lo que aprendieron a proyectos futuros. Estas actividades de evaluación posteriores al proyecto resultan útiles porque animan a los estudiantes a reflexionar,aumentando así la calidad de los resultados del aprendizaje.

Aunque la fase de planificación del curso es relativamente estructurado, los proyectos trabajados, los factores contextuales, y la combinación de estudiantes involucrados determina en última instancia las lecciones adicionales que han ido surgiendo hasta el momento.

Se valoran los trabajos en función de las competencias adquiridas, como son la adquisición significativa de contenidos, la responsabilidad por el propio aprendizaje, las habilidades de comunicación oral y escrita, el autoaprendizaje, el pensamiento reflexivo y las habilidades para el trabajo en equipo. Los resultados indican que este proyecto ha contribuido claramente a la mejora de la adquisición de todas las competencias, a excepción del pensamiento reflexivo, que parece que se ha reforzado en las asignaturas cursadas en su 
plan de estudios. Estos resultados nos sirven para continuar en esta línea de trabajo, puesto que confirman que realmente es posible lograr la implicación activa de los estudiantes y mejorar el aprendizaje.

\section{Responsabilidad social de los profesionales de la construcción}

En lo que concierne a la responsabilidad social universitaria en general, y dentro de la docencia en particular, capacitar a los docentes y a los estudiantes en las especialidades del Aprendizaje Basado en Proyectos, abriendo las aulas hacia la comunidad como fuente de enseñanza significativa y práctica aplicada a la solución de problemas reales es algo fundamental. Se buscan de cómo el estudiante puede aprender lo que tiene aprender formándose como ciudadano informado y responsable. Esto fomenta la creación de talleres de aprendizaje, mayor articulación entre las disciplinas (por la necesidad de un enfoque multi e interdisciplinario para tratar los proyectos), y mayor articulación entre la docencia, la investigación y la proyección social.

Se debe garantizar la responsabilidad social de la profesión de Arquitecto Técnico, y por extrapolación a todo lo que tiene relación con el mundo de la edificación. Debemos reconocer que, hasta ahora, después de siglos de desarrollo de los materiales de construcción, hemos fracasado en controlar el impacto ambiental y energético del sector, lo que nos ha conducido entre otros a los problemas ecológicos globales que todo el mundo conoce. Es por lo tanto necesario someter la actividad a un control social y político, entendiendo la responsabilidad individual y trasladándola a los profesionales del futuro, que son nuestros estudiantes del presente.

El lugar estratégico para instituir y promover la responsabilidad social de la ciencia y la tecnología, en todos sus ámbitos, es justamente la Universidad, porque es el lugar de convergencia entre la producción del saber científico (la investigación), la reproducción de este saber (transmisión de los conocimientos y formación de los profesionales que socializan las ciencias y tecnologías) y la información de la ciudadanía sobre los avances científicos y tecnológicos (la Universidad es un espacio social abierto al debate público).

La Universidad tiene la responsabilidad social de promover el debate, facilitarlo, conducirlo y enriquecerlo, dando al estudiante los medios para informarse, reflexionar y juzgar y a las empresas los conocimientos adecuados para aplicar su propia Responsabilidad Social. En este sentido, la aplicación de la investigación a la Titulación de Arquitectura Técnica, así como el trabajo pro proyectos, es una de las acciones que contribuyen a ese fin.

\section{Conclusiones}

Como conclusión principal se extrae la necesidad de implantar procesos de esta índole, y de asumir la esta práctica como objeto de análisis, reflexión e intervención, con responsabilidad ética y profesional. Más allá de la implantación de esta metodología en la asignatura y de los resultados de aprendizaje obtenidos, la respuesta estudiantil ha sido muy positiva. Es decir, el trabajo por proyectos ha tenido un impacto muy positivo en el 
rendimiento y los aprendizajes de los estudiantes así como el incremento de la satisfacción para con la experiencia educativa.

Por tanto, se debe favorecer el análisis de los resultados obtenidos para extraer conclusiones con los aspectos aplicables para la situación problema en una realidad contextual. El poder trabajar con criterios claros sobre la propia acción del estudiante, favorece la fundamentación científica, e impulsa la formación constante y permanente del buen hacer en el gremio profesional de los arquitectos técnicos.

\section{REFERENCIAS}

BROWN K.A. (2000). "Developing Project Management Skills: A Service Learning Approach". Project Management Institute, vol. 31, issue 4, p. 53-58.

GARCIA ARENAS C, CANO PALACIOS M, VEGA BORRERO F, LOUISE NILSSON S, VILCHES ARENAS L.F, LEIVA FERNANDEZ C. (2014) "Nueva metodología de aprendizaje autónomo aplicada a una práctica de Ingeniería Química mediante técnicas de investigación-acción". Revista d'innovació educativa, vol. 13, p. 97-103.

GARCÍA RUIZ R., GONZALEZ N, CONTRERAS P. (2014). "La formación en competencias en la universidad a través de proyectos de trabajo y herramientas 2.0. Análisis de una experiencia". Revista de Universidad y Sociedad del Conocimiento (RUSC). vol. 11, p.. 49-60.

KOKOTSAKI D, MENZIES V, WIGGINS A. (2016). "Project-based learning: A review of the literature". Improving Schools, vol. 19, issue 3, p. 267-277.

KINKUS J. (2007). "Project Management Skills: A Literature Review and Content Analysis of Librarian Position Announcements", College \& Research Libraries, vol. 363.

RUIZ G. (2002). "La sociedad del conocimiento y la educación superior universitaria". Revista Mexicana de Ciencias Políticas y Sociales, vol. 45, issue 185, p. 109-124.

SARIWATI M.S, ZAIMY J.J. NORINA A.J. (2013). "Assessment of Project Management Skills and Learning Outcomes in Students' Projects". Procedia - Social and Behavioral Sciences, vol. 90, p. $745-754$.

VITERI BRIONES T.A, VAZQUEZ CEDEÑO S. (2015). "Formación de habilidades de investigación formativa en los estudiantes de la carrera de ingeniería comercial de la Facultad de Ciencias Administrativas de la Universidad de Guayaquil". Revista Universidad y Sociedad, vol. 8, p. 36-44. 